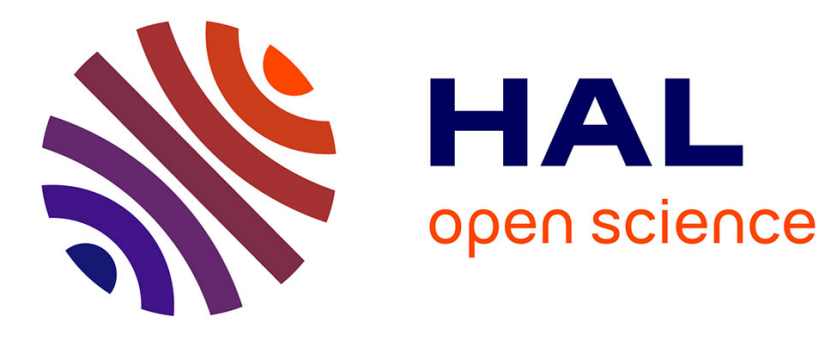

\title{
Dense disparity map representations for stereo image coding
}

\author{
Mounir Kaaniche, Wided Miled, Béatrice Pesquet-Popescu, Amel \\ Benazza-Benyahia, Jean-Christophe Pesquet
}

\section{To cite this version:}

Mounir Kaaniche, Wided Miled, Béatrice Pesquet-Popescu, Amel Benazza-Benyahia, Jean-Christophe Pesquet. Dense disparity map representations for stereo image coding. IEEE International Conference on Image Processing (ICIP'09), 2009, Le Caire, Egypt. pp.725-728, 10.1109/ICIP.2009.5414305 hal00621939

\section{HAL Id: hal-00621939 \\ https://hal.science/hal-00621939}

Submitted on 29 May 2013

HAL is a multi-disciplinary open access archive for the deposit and dissemination of scientific research documents, whether they are published or not. The documents may come from teaching and research institutions in France or abroad, or from public or private research centers.
L'archive ouverte pluridisciplinaire HAL, est destinée au dépôt et à la diffusion de documents scientifiques de niveau recherche, publiés ou non, émanant des établissements d'enseignement et de recherche français ou étrangers, des laboratoires publics ou privés. 


\title{
DENSE DISPARITY MAP REPRESENTATIONS FOR STEREO IMAGE CODING
}

\author{
M. Kaaniche ${ }^{1,2}$,W. Miled ${ }^{1}$, B. Pesquet-Popescu ${ }^{1}$, A. Benazza-Benyahia ${ }^{2}$ and J.- C. Pesquet ${ }^{3}$ \\ ${ }^{1}$ Telecom ParisTech, \\ Signal and Image Proc. Dept. \\ 37-39, rue Dareau, 75014 Paris, France \\ kaaniche@telecom-paristech.fr \\ pesquet@telecom-paristech.fr \\ miled@telecom-paristech.fr \\ ${ }^{2}$ SUP'COM, URISA \\ Cité Technologique des \\ Communications, 2083, Tunisia \\ benazza.amel@supcom.rnu.tn \\ ${ }^{3}$ Université Paris-Est, \\ IGM and UMR-CNRS 8049, \\ Champs-sur-Marne, 77454 \\ Marne-la-Vallée, France \\ pesquet@univ-mlv.fr
}

\begin{abstract}
Research in stereo image coding has focused on the disparity estimation/compensation process to exploit the cross-view redundancies. Most of the reported methods use a classical block-based technique in order to estimate the disparity field. However, this estimation technique does not always provide an accurate disparity map, which may affect the disparity compensation step. In this paper, we propose to use an estimation method that produces a dense and smooth disparity map. Then, on the one hand, this map is segmented and efficiently coded by exploiting the high correlation between neighboring disparity values. On the other hand, we integrate the disparity information into a vector lifting scheme for stereo image coding. Experimental results indicate that the proposed coding scheme outperforms the conventional methods employing a block-based disparity estimation.
\end{abstract}

Index Terms - disparity estimation, dense disparity map, stereoscopic image coding, vector lifting scheme.

\section{INTRODUCTION}

A stereoscopic imaging system consists of two cameras recording the same scene from two slightly different view points. By presenting the appropriate image of a stereo pair to the left/right eye, the viewer perceives the scene in three dimensions. The continuous advances in acquisition and display technologies have entailed the widespread development of stereovision applications in various fields such as telepresence in videoconferences [1], computer vision and remote sensing. The increasing interest in stereo image applications has led to a growing demand for efficient compression techniques for both storage and transmission purposes. As the two images have similar content, efficient coding schemes have been designed to exploit the cross-view redundancies $[2,3]$. This is achieved by estimating the spatial displacement between the left and right images of the stereo pair [4], leading to the computation of the so-called disparity map. The Disparity Estimation problem (DE) has been extensively studied in computer vision and surveys of the different techniques proposed in the literature can be found in $[5,6]$. Traditionally, disparity estimation algorithms are basically classified into two categories: block-based approaches and pixel-based ones. In the first category, it is assumed that the disparity is blockwise constant and the objective is to find the best matching block. However, this assumption does not always hold, especially around depth discontinuities and in textureless regions. Pixel-based approaches attempt to overcome this drawback by assigning a disparity value to each point visible in the stereo pair. Once the disparity map is available, the most frequently reported methods for stereo image coding proceed as follows: first, the reference image (say the left one) is independently encoded (as an intra-image), then the other image (target) is predicted from the reference, using the estimated disparity map. Finally, the disparity map, the reference image and the prediction error image are encoded. This approach is known as disparity compensation, due to its similarity with motion compensation techniques which are widely employed in video coding [7]. It is worth pointing out that the disparity field is generally losslessly encoded using DPCM with arithmetic coding [1, 2, 3] whereas the reference and the residual images are coded in different transform domains. For instance, some methods make use of the discrete cosine transform $[8,9]$, and to meet the scalability requirement, more recent works have retained the wavelet transform $[10,11]$. Recently, we have proposed a novel approach based on the Vector Lifting Schemes (VLS), that directly generates two compact multiresolution representations of the left and right images instead of a residual image [12]. Inspired by the works in video coding, the disparity field was estimated based on a block-matching technique. As previously mentioned, the limitation of this technique is that it fails at object edges and boundaries. Besides, if the block size becomes smaller, the overhead required to transmit the disparity information becomes too large w.r.t. the overall bitrate. Further improvement can be achieved by employing variable block size [13] and resorting to rate-distortion based algorithms similar to those employed in the framework of motion-compensated video coding [14]. However, the classical block-based technique does not always guarantee a consistent disparity map.

In this paper, we propose to perform the joint coding of the stereo pair using the VLS developed in [12] along with the Dense DE (DDE) method described in $[15,16]$. We have retained such DE method because it achieves good results compared with the state-ofthe-art methods, such as dynamic programming [17] and graph cuts [18]. Based on a set theoretic framework, this DDE approach incorporates various convex constraints corresponding to a priori information and yields disparity vectors with ideally infinite precision. A total variation based regularization constraint is considered in order to output a smooth disparity field while preserving discontinuities. The coding of the resulting dense disparity map is a challenging issue because of its contribution to the final bitrate. One novelty of this paper is to resort to an efficient quadtree decomposition and entropy coding of the generated disparity map that achieve a tradeoff between the accuracy of the disparity representation and the coding cost.

The remainder of this paper is organized as follows. In Sec- 
tion 2, we recall the principles of our joint coding scheme based on VLS. In Section 3, we describe the retained DDE method. We also address the problem of a suitable representation of the disparity map for coding purposes. Finally, in Section 4, experimental results are given and some conclusions are drawn in Section 5.

\section{VLS BASED DECOMPOSITION}

Recently, we have proposed a new joint coding scheme for stereo pairs using a VLS [12]. In fact, instead of coding the reference image and the residual one, our approach is based on a joint multiscale decomposition of the left and the right image. It consists of coding the reference image $I^{(l)}$ in intra mode whereas $I^{(r)}$ is coded according to a hybrid mode driven by the available disparity map. More precisely, the conventional 5/3 decomposition (a predict-update, $\mathrm{P}$ $\mathrm{U}$, structure) is applied to the reference image $I^{(l)}[19]$. At each resolution level $(j+1)$, the related detail coefficients $\widetilde{d}_{j+1}^{(l)}$ and the approximation coefficients $\widetilde{I}_{j+1}^{(l)}$ are expressed as follows:

$$
\begin{aligned}
& \widetilde{d}_{j+1}^{(l)}(x, y)=I_{j}^{(l)}(x, 2 y+1)-\left\lfloor\frac{1}{2}\left(I_{j}^{(l)}(x, 2 y)+I_{j}^{(l)}(x, 2 y+2)\right)\right\rfloor \\
& \widetilde{I}_{j+1}^{(l)}(x, y)=I_{j}^{(l)}(x, 2 y)+\left\lfloor\frac{1}{4}\left(d_{j+1}^{(l)}(x, y)+d_{j+1}^{(l)}(x, y+1)\right)\right\rfloor .
\end{aligned}
$$

For the target image $I^{(r)}$, we use a P-U-P structure. It incorporates a second prediction step which involves a hybrid prediction exploiting at the same time the intra and inter-image redundancies in the stereo pair. This is achieved by using the estimated disparity field denoted by $\mathbf{v}=\left(v_{x}, v_{y}\right)$. Therefore, the VLS based decomposition is described as follows:

$$
\begin{aligned}
& \widetilde{d}_{j+1}^{(r)}(x, y)=I_{j}^{(r)}(x, 2 y+1)-\left\lfloor\frac{1}{2}\left(I_{j}^{(r)}(x, 2 y)+I_{j}^{(r)}(x, 2 y+2)\right)\right\rfloor \\
& \widetilde{I}_{j+1}^{(r)}(x, y)=I_{j}^{(r)}(x, 2 y)+\left\lfloor\frac{1}{4}\left(d_{j+1}^{(r)}(x, y)+d_{j+1}^{(r)}(x, y+1)\right)\right\rfloor \\
& \widetilde{d}_{j+1}^{(r)}(x, y)=\widetilde{d}_{j+1}^{(r)}(x, y)-\left\lfloor q_{j}\left(\widetilde{I}_{j+1}^{(r)}(x, y)+\widetilde{I}_{j+1}^{(r)}(x, y+1)\right)\right. \\
& \left.+\sum_{k \in \mathcal{P}_{j}^{(r, l)}} p_{j, k}^{(r, l)} I_{j}^{(l)}\left(x+v_{x, j}(x, 2 y+1), 2 y+1+v_{y, j}(x, 2 y+1)-k\right)\right\rfloor
\end{aligned}
$$

where $\left(v_{x, j}, v_{y, j}\right)$ is obtained by subsampling and dividing by $2^{j}$ the initial full resolution estimated disparity vector $\left(v_{x}, v_{y}\right)$, since the dimensions of the subbands at the $j$-th resolution level correspond to the dimensions of the initial images divided by $2^{j}$. The set $\mathcal{P}_{j}^{(r, l)}$ denotes the spatial support of the inter-image predictor and $p_{j, k}^{(r, l)}$ (resp. $q_{j}$ ) correspond to the weights of the inter (resp. intra) image predictor. In addition, at the last resolution level $j=J$, instead of directly coding the approximation $I_{J}^{(r)}$, we predict it from the approximation subband at the last resolution level of the left image. As a result, the following residual subband $e_{J}^{(r)}$ is generated:

$$
e_{J}^{(r)}(x, y)=I_{J}^{(r)}(x, y)-\left\lfloor p_{J}^{(r, l)} I_{J}^{(l)}\left(x+v_{x, J}(x, y), y+v_{y, J}(x, y)\right)\right\rfloor .
$$

It is expected that $e_{J}^{(r)}$ can be coded more economically than $I_{J}^{(r)}$. The coefficients $q_{j}$ and $p_{j, k}^{(r, l)}$ (resp. $p_{J}^{(r, l)}$ ) are optimized at each resolution level $j<J$ (resp. at the coarsest resolution level $J$ ) by minimizing the variance of the prediction error $\breve{d}_{j+1}^{(r)}\left(\right.$ resp. $\left.e_{J}^{(r)}\right)$.

\section{PROPOSED DISPARITY ESTIMATION AND CODING METHOD}

\subsection{Retained DDE procedure}

In this section, the stereo images are assumed to be geometrically rectified. Hence, the disparity field is restricted to the horizontal component (i.e $\mathbf{v}=\left(v_{x}, 0\right)$ ). DDE methods aim at assigning a disparity value $\hat{v}_{x}$ to every pixel $(x, y)$ in $I^{(r)}$ so that the resulting field minimizes a given cost functional $\tilde{J}$. Generally, the chosen criterion $\tilde{J}$ is the sum of squared intensity differences:

$$
\begin{aligned}
& \left(\hat{v}_{x}(x, y)\right)_{(x, y) \in \mathcal{D}}=\arg \min _{v_{x} \in \Omega} \tilde{J}\left(v_{x}\right), \\
= & \arg \min _{v_{x} \in \Omega} \sum_{(x, y) \in \mathcal{D}}\left[I^{(r)}(x, y)-I^{(l)}\left(x+v_{x}(x, y), y\right)\right]^{2}
\end{aligned}
$$

where $\mathcal{D}$ is the image support and $\Omega$ is the range of candidate disparity values. Generally, an initial coarse estimate $\bar{v}_{x}$ of $v_{x}$ is made available, say thanks to a correlation based-method. Assuming that the magnitude difference of both fields is relatively small, the warped left image around $\bar{v}_{x}$ is approximated by a Taylor expansion:

$$
I^{(l)}\left(x+v_{x}, y\right) \simeq I^{(l)}\left(x+\bar{v}_{x}, y\right)+\nabla I_{x}^{(l)}\left(x+\bar{v}_{x}, y\right)\left(v_{x}-\bar{v}_{x}\right)
$$

where $\nabla I_{x}^{(l)}\left(x+\bar{v}_{x}, y\right)$ is the horizontal gradient of the warped left image. Note that in (2), we have not made explicit that $v_{x}$ and $\bar{v}_{x}$ are functions of $s=(x, y)$ for notation concision. Using the linearization (2), the criterion $\tilde{J}$ in (1) can be approximated by a functional $J$ which is quadratic convex in $v_{x}$ :

$$
J\left(v_{x}\right)=\sum_{s \in \mathcal{D}}\left[r(s)-L(s) v_{x}(s)\right]^{2} .
$$

where

$$
\begin{aligned}
& L(s)=\nabla I_{x}^{(l)}\left(x+\bar{v}_{x}(s), y\right) \\
& r(s)=I^{(r)}(s)-I^{(l)}\left(x+\bar{v}_{x}(s), y\right)+\bar{v}_{x}(s) L(s) .
\end{aligned}
$$

Minimizing this quadratic objective function is an ill-posed problem as the components of $L$ may locally vanish. Thus, to convert this problem to a well-posed one, it is used to incorporate additional constraints reflecting the prior knowledge about the disparity field. In this work, we address the problem through a set theoretic framework. Firstly, each constraint is represented by a closed convex set $S_{m}$ with $m \in\{1, \ldots, M\}$, in a Hilbert image space $\mathcal{H}$. The intersection $S$ of all the $M$ sets $\left(S_{m}\right)_{1 \leq m \leq M}$ constitutes the family of possible solutions $[15,16]$. Therefore, it is enough to find an acceptable solution in $S$ which minimizes the underlying objective function $J$ :

$$
\text { Find } \hat{v}_{x} \in S=\bigcap_{m=1}^{M} S_{m} \text { such that } J\left(\hat{v}_{x}\right)=\inf _{v_{x} \in S} J\left(v_{x}\right) .
$$

The constraint sets can be modeled as level sets:

$$
\forall m \in\{1, \ldots, M\}, \quad S_{m}=\left\{v_{x} \in \mathcal{H} \mid f_{m}\left(v_{x}\right) \leq \delta_{m}\right\}
$$

where $f_{m}: \mathcal{H} \rightarrow \mathbb{R}$ is a continuous convex function for every $m \in\{1, \ldots, M\}$ and $\left(\delta_{m}\right)_{1 \leq m \leq M}$ are real-valued parameters such that $S=\bigcap_{m=1}^{M} S_{m} \neq \emptyset$.

Hence, it is required to define the convex sets $\left(S_{m}\right)_{1 \leq m \leq M}$ to proceed to the DDE procedure within the set theoretic framework. At this level, it is important to emphasize the great flexibility in incorporating any set of arbitrary convex constraints. In what follows, 
we will focus on $M=3$ constraints. The first one consists of restricting the variation of the disparity $v_{x}$ within a specified range $\left[v_{\min }, v_{\max }\right]$. This can be expressed by the following constraint set $S_{1}$ :

$$
S_{1}=\left\{v_{x} \in \mathcal{H} \mid v_{\min } \leq v_{x} \leq v_{\max }\right\} .
$$

Most importantly, a constraint can be incoporated in order to strengthen the smoothness of the disparity field in the homogeneous areas while preserving edges. Indeed, neighboring pixels belonging to the same object should have similar disparities. This can be achieved by considering the total variation $\operatorname{tv}\left(v_{x}\right)$ which can be defined as the sum over $\mathcal{D}$ of the norm of the spatial gradient of $v_{x}$ [20]. More precisely, a total variation based regularization constraint amounts to impose an upper bound $\tau$ on tv:

$$
S_{2}=\left\{v_{x} \in \mathcal{H} \mid \operatorname{tv}\left(v_{x}\right) \leq \tau\right\} .
$$

It is worth pointing out that the positive constant $\tau$ can be estimated through a learning procedure on image databases [21]. Moreover, an oriented smoothness constraint aims at preserving the discontinuities corresponding to the edges of the right image $I^{(r)}$. By using the Nagel-Enkelmann operator $D[22,23]$, it amounts to introduce $\left(\nabla v_{x}(s)\right)^{\top} D\left(\nabla I^{(r)}(s)\right)\left(\nabla v_{x}(s)\right)$ as a regularization term:

$$
S_{3}=\left\{v_{x} \in \mathcal{H} \mid \sum_{s \in \mathcal{D}}\left(\nabla v_{x}(s)\right)^{\top} D\left(\nabla I^{(r)}(s)\right)\left(\nabla v_{x}(s)\right) \leq \kappa\right\}
$$

where the positive parameter $\kappa$ may be approximated from prior experiments (see Section 4). Finally, the disparity estimation problem is formulated by minimizing the quadratic objective function $J$ in (3) under the $M=3$ mentioned constraint sets $\left(S_{m}\right)_{1 \leq m \leq 3}$. Among the existing optimization algorithms, we have retained the efficient constrained quadratic minimization technique developed in [24] which is adapted to convex problems with quadratic objective functions. Due to space limitation, we will not describe the algorithm but for more details, the reader is referred to [24, 15].

\subsection{Dense disparity map coding}

Concerning coding applications, the proposed DDE raises an important issue: the expensive cost of transmission/storage of the disparity map in terms of bitrate [25]. In order to reduce this cost, we propose to apply a quadtree decomposition (QTD) to the estimated field $\hat{v}_{x}$. Thus, the map $\hat{v}_{x}$ is segmented into macroblocks $\mathcal{B}^{\left(\hat{v}_{x}\right)}$ of size $b_{x} \times b_{y}$ (typically, $16 \times 16$ ). The homogeneity of $\mathcal{B}^{\left(\hat{v}_{x}\right)}$ is measured by its local variance $\sigma_{\mathcal{B}}^{2}$. Thus, for a given threshold value $T$, if $\sigma_{\mathcal{B}}^{2} \leq T$, all the disparity values within $\mathcal{B}^{\left(\hat{v}_{x}\right)}$ are replaced by a single disparity value: it may be the average or the median value. At the opposite, if $\sigma_{\mathcal{B}}^{2}>T, \mathcal{B}^{\left(\hat{v}_{x}\right)}$ is subdivided into four subblocks and then the test of homogeneity is recursively repeated for each subblock. Therefore, homogeneous areas will be represented by larger blocks, whereas small blocks will correspond to object boundaries or occluded regions. It is worth pointing out that the segmented disparity map and the mode information corresponding to the block size assigned to each disparity value must be transmitted as side information. In our experiments, we have employed the encoding module integrated in the H.264 JM software based on DPCM technique with median predictors and variable-length codes.

\section{EXPERIMENTAL RESULTS}

Simulation results are performed on the three real stereo pairs: "Shrub," "Outdoor" and "Book Arrival". For the block-based disparity estimation method (BDE), we have used the displacement estimation module in H. 264 JM software based on variable size block-matching. For the proposed DDE, the minimal and maximal values of the disparity amplitudes $v_{\min }$ and $v_{\max }$ were set by measuring the disparity at certain points of interest. The bounds $\tau$ and $\kappa$ related to constraints $S_{2}$ and $S_{3}$ are obtained by first calculating the value of the associated convex functions on the initial disparity field $\bar{v}_{x}$ and then choosing only a given ratio (e.g. 20\%) of these values. The coding module in the H.264 JM is applied to each of the resulting maps, the delivered bitrates being denoted by $R^{\left(v_{x}\right)}$. Figure 1 shows the target image and the disparity maps provided by the BDE and the DDE. It is obvious that the DDE method generates the smoothest map. Furthermore, the resulting bitrate $R^{\left(v_{x}\right)}$ for the dense map is 50 times greater than the bitrate of the block-based map. However, by exploiting the smoothness of the dense map owing to a QTD with median filtering (DDE+QTD) followed by a rounding operation, $R^{\left(v_{x}\right)}$ is dramatically reduced. The corresponding disparity map is displayed in Fig. 1(d): it is more robust to noise estimation compared with the BDE. In addition, Table 1 gives the bitrate $R^{\left(v_{x}\right)}$ of the disparity map and the PSNR of the disparitycompensated image between the three methods. It can be noticed that the proposed method reduces the bitrate of the disparity map up to $0.02 \mathrm{bpp}$ without sacrificing the disparity compensated image quality. In order to emphasize the benefit from using the DDE, two stereo image coding schemes are considered. The first one is the basic algorithm which consists of coding the left and the residual images. It will be designated by scheme-B [11]. The second one is our VLS described in Section 2. Fig. 2 illustrates the scalability in quality of the reconstruction procedure by providing the PSNR versus the bitrate for the stereo pair "Shrub". It is worth pointing out that our DDE method outperforms the classical BDE method by $0.1-0.6 \mathrm{~dB}$ for our VLS (resp. by $0.1-0.5 \mathrm{~dB}$ for the scheme-B) whatever the employed decomposition scheme of the stereo pair. The proposed method inherits the quality scalability properties of the JPEG2000 codec, preserved through the joint multiresolution analysis. Our stereo still image coding scheme based on VLS is a lossy-to-lossless technique in which the disparity map is estimated only once, and from this point of view it differs from existing stereo video schemes based on H.264 coding tools (including stereo SEI message, for example, [26]), where the disparity is estimated for different bitrates (i.e for each point of the PSNR curve). When considering a stereo video coding framework, a comparison with H.264 standard can be found in [27].

\section{CONCLUSION}

In this paper, we have presented the benefits of using a dense disparity map in stereo image coding schemes. Unlike conventional methods that employ a block-based disparity estimation method, our method uses a dense and smooth disparity map. Experimental results showed that our approach produces better results than classical methods. Ongoing research aims at extending this stereo image coding scheme to multi-view image and stereo video coding applications.

Table 1. Disparity map representations in terms of bitrate (bpp) and PSNR $(\mathrm{dB})$ of the disparity compensated image.

\begin{tabular}{|c|l|l|l|l|l|l|}
\cline { 2 - 7 } \multicolumn{1}{c|}{} & \multicolumn{2}{c|}{ BDE } & \multicolumn{2}{c|}{ DDE } & \multicolumn{2}{c|}{ DDE+QTD } \\
\cline { 2 - 7 } & $R^{\left(v_{x}\right)}$ & PSNR & $R^{\left(v_{x}\right)}$ & PSNR & $R^{\left(v_{x}\right)}$ & PSNR \\
\hline Book & 0.058 & 29.60 & 3.80 & 33.54 & 0.035 & 30.40 \\
\hline Shrub & 0.061 & 34.25 & 3.01 & 35.30 & 0.041 & 34.29 \\
\hline Outdoor & 0.075 & 30.32 & 3.88 & 30.98 & 0.058 & 30.38 \\
\hline
\end{tabular}




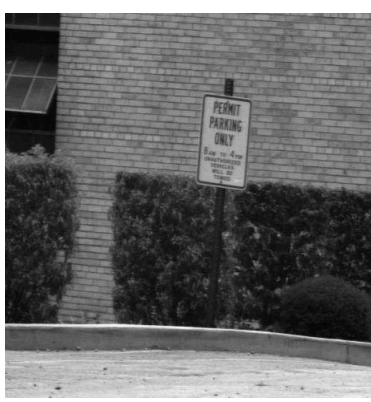

(a) Right image

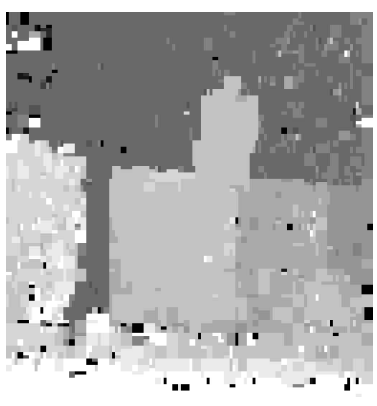

(b) $R^{\left(v_{x}\right)}=0.061 \mathrm{bpp}$

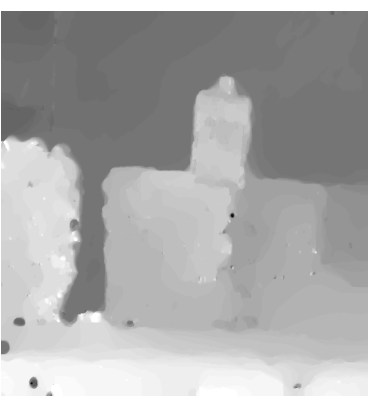

(c) $R^{\left(v_{x}\right)}=3.01 \mathrm{bpp}$

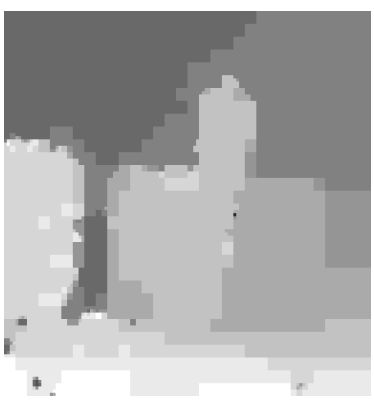

(d) $R^{\left(v_{x}\right)}=0.041 \mathrm{bpp}$

Fig. 1. Extracted disparity maps for the Shrub right image using (b) a block-based DE method (c) the proposed DDE method (d) a quadtree decomposition of the computed dense disparity map $(T=0.2)$.

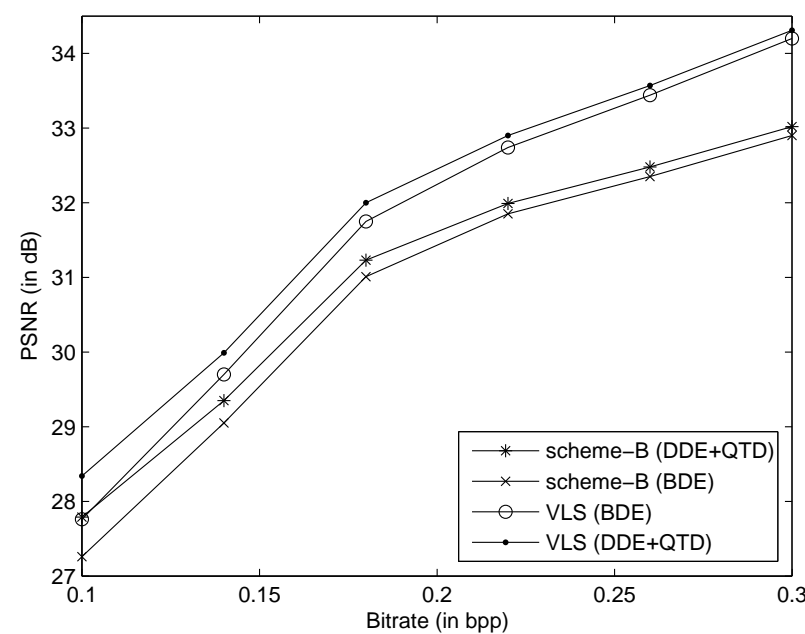

Fig. 2. PSNR (in dB) versus the bitrate (bpp) after JPEG2000 encoding for the stereo pair "shrub".

\section{REFERENCES}

[1] M. Waldowski, "A new segmentation algorithm for videophone applications based on stereo image pairs," IEEE Trans. on Communications, vol. 39, no. 12, pp. 1856 1868, December 1991

[2] T. Frajka and K. Zeger, "Transform coding of stereo image compression," Optical Engineering, vol. 42, no. 1, pp. 182-189, January 2003

[3] J. N. Ellinas and M. S. Sangriotis, "Morphological wavelet-based stereo image coders," Journal of Visual Communication and Image Representation, vol. 17, no. 4, pp. 686-700, August 2006.

[4] N. Grammalidis and M. G. Strintzis, "Disparity and occlusion estimation in multiocular systems and their coding for the communication of multiview image sequences," IEEE Trans. on Circuits and Systems for Video Technology, vol. 8, no. 3, pp. 328-344, June 1998 .

[5] D. Scharstein and R. Szeliski, "A taxonomy and evaluation of dense two-frames stereo correspondance algorithms," Internat. Journal of Computer Vision, vol. 47 , no.1, pp. 7-42, June 2002.

[6] M. Z. Brown, D. Burschka and G. D. Hager, "Advances in computational stereo," IEEE Trans. on Pattern Analysis and Machine Intelligence, vol. 25, no. 8, pp. 993-1008, August 2003.

[7] S. Wang and H. Chen, "An improved algorithm of motion compensation MPEG video compression," Internat. Vehicle Electronics Conf., vol. 1, pp. 261-264, Changchun, China, September 1999.

[8] J. Han and Z. Lu, "DCT-based embedded coding scheme for stereo image," In ternat. Conf. on Communications, Circuits and Systems, vol. 1, pp. 543-547, Chengdu, China, June 2004

[9] M. S. Moellenhoff and M. W. Maier, "Transform coding of stereo image residuals," IEEE Trans. on Image Processing, vol. 7, no. 6, pp. 804-812, June 1998.
[10] J. Xu, Z. Xiong and S. Li, "High performance wavelet-based stereo image coding," IEEE Internat. Symposium on Circuits and Systems, vol. 2, pp. 273-276, PhoenixScottsdale, AZ, USA, May 2002.

[11] N. V. Boulgouris and M. G. Strintzis, "A family of wavelet-based stereo image coders," IEEE Trans. on Circuits and Systems for Video Technology, vol. 12, no. 10, pp. 898-903, October 2002.

[12] M. Kaaniche, A. Benazza-Benyahia, B. Pesquet-Popescu and J.-C. Pesquet, "Vector lifting schemes for stereo image coding," to appear in IEEE Transactions on Image Processing, 2009.

[13] W. Woo and A. Ortega, "Overlapped block disparity compensation with adaptive windows for stereo image coding," IEEE Trans. on Circuits and Systems for Video Technology, vol. 10, no. 2, pp. 194-200, March 2000.

[14] Joint Draft 5.0 on Multiview Video Coding, JVT-Y209, Shenzhen, China, October 2007.

[15] W. Miled, J.-C. Pesquet and M. Parent, "Disparity map estimation using a total variation bound," Canadian Conf. Computer Robot Vision, Quebec, Canada, pp. 48-55, June 2006.

[16] W. Miled, J.-C. Pesquet and M. Parent, "A convex optimization approach for depth estimation under illumination variation," IEEE Transactions on Image Processing, vol. 18, pp. 813-830, April 2009.

[17] O. Veksler, "Stereo correspondence by dynamic programming on a tree," Int. Conf. Comput. Vis. Pattern Recognit., San-Diego, CA, USA, vol. 2, pp. 384-390, June 2005.

[18] M. Bleyer and M. Gelautz, "Graph-based surface reconstruction from stereo pairs using image segmentation," Proc. of SPIE, Videometrics VIII, San Jose, CA, USA, vol. 5665, pp. 288-299, January 2005.

[19] W. Sweldens, "The lifting scheme: a new philosophy in biorthogonal wavelet constructions," Proceedings of SPIE, San-Diego, CA, USA, 2569, pp. 68-79, September 1995.

[20] L. I. Rudin, S. Osher, and E. Fatemi, "Nonlinear total variation based noise removal algorithms," Physica D, vol. 60, pp. 259-268, 1992.

[21] P. L. Combettes and J.-C. Pesquet, "Image restauration subject to a total variation constraint," IEEE Transactions on Image Processing, no. 9, vol. 13, pp. 1213 1222, September 2004

[22] H. H. Nagel and W. Enkelmann, "An investigation of smoothness constraints for the estimation of displacement vector fields from image sequences," IEEE Trans. on Pattern Analysis and Machine Intelligence, pp. 565-593, 1987.

[23] L. Alvarez, R. Deriche, J. Sanchez, and J. Weickert, "Dense disparity map estimation respecting image discontinuities: A pde and scale-space based approach," Journal of Visual Communication and Image Representation, vol. 13, pp.3-21, 2002

[24] P. L. Combettes, "A block iterative surrogate constraint splitting method for quadratic signal recovery," IEEE Trans. on Signal Processing, vol. 51, no. 7, pp. 1771-1782, July 2003.

[25] I. Daribo, C. Tillier, B. Pesquet-Popescu,"Adaptive wavelet coding of the depth map for stereoscopic view synthesis," International Workshop on Multimedia Signal Processing, pp. 413-417, Cairns, Australia, October 2008.

[26] S. Sun and S. Lei,"Stereo-view video coding using H. 264 tools," Proc. of SPIE Image and video communications and processing, vol. 5685, pp. 177-184, March 2005.

[27] I. Daribo, M. Kaaniche, W. Miled, M. Cagnazzo and B. Pesquet-Popescu,"Dense disparity estimation in multiview video coding," International Workshop on Multimedia Signal Processing, Rio de Janeiro, Brazil, October 2009. 